\title{
Horizontal spatial correlation of reverberation for rough sea-bottom interface
}

\author{
Shanyong Yan ${ }^{1,2}$, Changhong Wang ${ }^{1,3, *}$ \\ ${ }^{1}$ Ocean Acoustic Technology Center, Institute of Acoustics, CAS, 100190 Beijing, China \\ ${ }^{2}$ University of Chinese Academy of Sciences, 100049 Beijing, China \\ ${ }^{3}$ Beijing Engineering Technology Research Center of Ocean Acoustic Equipment, 100190 Beijing, China
}

\begin{abstract}
Correlation sonar, which estimates the velocity of vessel utilizing the principle of waveform invariance, can achieve the sampling of the horizontal spatial correlation of sea-bottom reverberation. The horizontal spatial correlation can be expressed as a correlation function and is affected by sea-bottom characteristics. The expression of the correlation function of the sea-bottom reverberation is derived, which is written as the convolution of the autocorrelation function of transmitted signal, the cross-correlation function of the backscattered impulse response from a plane interface, and the autocorrelation function of the probability density function of the sea-bottom roughness. The isotropic interface roughness of the sea-bottom leads to a circular planform of the correlation function whose width varies with roughness. The anisotropic interface roughness of the sea-bottom leads to an elliptical planform of the correlation function whose major axis is in the direction of weaker roughness. Simulation of submarine reverberation and correlation function verifies this conclusion. The model for the spatially covariant field is used to estimate the backscattering cross section which varies with azimuth angle under the condition of anisotropic seafloor roughness. It should be noted that the horizontal spatial correlation of reverberation is also related to sonar parameters and other sea-bottom characteristics.
\end{abstract}

\section{Introduction}

The acoustic correlation $\log$ (ACL) estimates the velocity of vessel by matching the theoretical and the data's correlation function. The theoretical correlation function is the result of modelling the horizontal spatial correlation of sea-bottom reverberation, the data's correlation function is obtained by the correlation of signals received by receivers in different positions of a plane[1-4]. The horizontal spatial correlation of the sea-bottom rever-beration is inevitably affected by the characteristics of the sea-bottom. Briggs et al. measured the rough heights of the sea-bottom in the continental shelf and analyzed the one-dimensional roughness spectrum. The discrepancy in the roughness spectrum of different directions shows the anisotropy of the sea-bottom roughness[5,6]. This discrepancy is more pronounced on the two-dimensional power spectrum of the sea-bottom. Lyons et al. used a digital photogrammetry system to obtain two-dimensional digital elevation models (DEM) and two-dimensional power spectrums[7,8]. Jackson et al. modeled the interface roughness spectrum of the sea-bottom and analyzed the causes and laws of the evolution of the sea-bottom over time $[9,10]$. Ma et al. analyzed the signal backscattered from small roughness sea-bottom, and expressed the statistical average of the backscattered impulse response as a convolution between the probability density function of the sea-bottom roughness and the backscattered impulse response from a plane interface[11].
Smith and Atkins interpreted the scattering process as the role of a deep, random phase screen and analyzed the horizontal spatial correlation[12]. Restrepo and Mcdaniel derived an expression for the spatial covariance of the scattered field[13]. Under the circumstance of anisotropic sea-bottom roughness, Premus and Alexandrou used this expression to obtain the anisotropic sea-bottom backscattered intensity[14].

In this paper, the expression of the correlation function has been derived, which can reveal the effect of the sea-bottom interface roughness on the horizontal spatial correlation of the reverberation. This conclusion is verified by simulating the reverberation and its correlation function.

\section{The model of sea-bottom interface roughness}

In general, storms and strong tidal currents lead to anisotropic sea-bottom roughness. In the absence of hydrodynamic forcing by them, small scale, random sediment transport caused by biological activity occupies an important position. Jackson regards this biological process as a diffusive process which dominates the evolution of sea-bottom roughness features, and ultimately results in the isotropy of the sea-bottom roughness.

The isotropic interface roughness of the sea-bottom can be characterized by a one-dimensional roughness

Corresponding author: wangch@mail.ioa.ac.cn 
power spectrum (roughness spectra) in the form of power-law

$$
S\left(\mathbf{K}_{1}\right)=w_{2} k^{-\gamma_{2}}
$$

where $w_{2}$ is the spectral intensity, $\gamma_{2}$ is the spectral exponent, $\mathbf{K}_{1}$ is a two-dimensional wave vector with magnitude equal to the wavenumber $k$. The one-dimensional roughness spectrums in different directions of the same location can reflect the anisotropy of sea-bottom roughness. The two-dimensional roughness spectrum, which is more than the superposition of one-dimensional roughness spectrums in all directions, can more intuitively reflect the anisotropy of the sea-bottom roughness. According to the model of the sea-bottom roughness spectrum, an anisotropic rough sea-bottom can be obtained[15]

$$
S\left(\omega_{x}, \omega_{y}\right)=\sigma^{2} I\left(\omega_{x}, \omega_{y}\right)^{2(1-d)} A_{\alpha, \varphi}\left(\omega_{x}, \omega_{y}\right)^{-2}
$$

where

$$
\begin{aligned}
I\left(\omega_{x}, \omega_{y}\right)= & \sin ^{2}\left(\omega_{x} / 2\right)+\sin ^{2}\left(\omega_{y} / 2\right) \\
A_{\alpha, \varphi}\left(\omega_{x}, \omega_{y}\right)= & (1-\alpha \cos \varphi) \sin ^{2}\left(\omega_{x} / 2\right) \\
& +(1+\alpha \cos \varphi) \sin ^{2}\left(\omega_{y} / 2\right) \\
& -(1 / 2) \alpha \sin \varphi \sin \omega_{x} \sin \omega_{y}
\end{aligned}
$$

represent the isotropic part and the anisotropic part of the roughness spectrum respectively. In this expression, $\sigma$ is the rms height. The fractional parameter $0<d<1 / 2$ determines the degree of sea-bottom roughness. The closer $d$ to $1 / 2$, the smoother the sea-bottom. The anisotropy coefficient $0<\alpha<1$ characterizes the disper-sion of the sea-bottom roughness spectrum around this privileged direction. In particular, for $\alpha=0$, we find an isotropic model. $0 \leq \varphi<2 \pi$ determines the orientation of the roughness spectrum. In the case of determining the four parameters, the seafloor roughness spectrum can be determined. If the sea-bottom has a uniform random phase, the DEM of the sea-bottom can be recovered. Fig.1 and Fig.2 show the anisotropic sea-bottom roughness spectrum and the DEM of the sea-bottom recovered from it, respectively. The DEM of the sea-bottom does not exhibit anisotropy, but it can be seen that the roughness spectrum shows significant differences in two mutually perpendicular directions. In fact, the DEM will exhibit anisotropic ripple characteristics when $\alpha$ is close to 1 .

\section{The horizontal spatial correlation of sea-bottom reverberation}

Assuming that the processes of signal propagation and submarine scattering are linear systems, it has the relationship[11]

$$
y(t)=s(t) * m(t) * T(t) * h(t)
$$

where * denotes the convolution and $y(t)$ is the received signal, $s(t)$ is the emitted signal, $m(t)$ is the propagation function, $T(t)$ is the transducer's transfer function, and $h(t)$ is the sea-bottom impulse response. Ignoring $m(t)$ and $T(t)$, then

$$
y(t)=s(t) * h(t)=s(t) * h_{p}(t) * f
$$

where $h_{p}(t)$ is the backscattered impulse response from a plane interface, $f$ is the probability density function of the sea-bottom roughness.

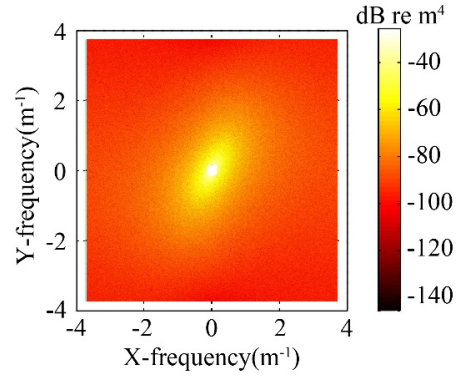

Fig. 1. sea-bottom roughness spectrum generated using the parameters $\sigma=0.0125, \alpha=0.6, \varphi=60^{\circ}, d=0.3$.

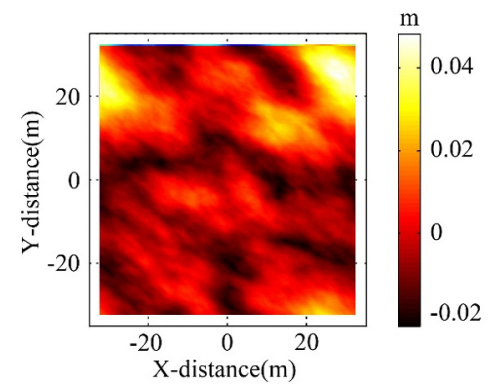

Fig. 2. DEM of the sea-bottom recovered from Fig.1.

Assuming that the array of the correlation sonar comprises a transmitting transducer and a plurality of hydrophones. Two signals received by two hydrophones at different locations are as follows

$$
\begin{gathered}
y_{1}\left(t_{1}, d_{1}\right)=s\left(t_{1}, d_{1}\right) * h_{p 1}\left(t_{1}, d_{1}\right) * f \\
y_{2}\left(t_{2}, d_{2}\right)=s\left(t_{2}, d_{2}\right) * h_{p 2}\left(t_{2}, d_{2}\right) * f
\end{gathered}
$$

The correlation of the two signals can be expressed as

$$
\begin{aligned}
R\left(\tau, \mathbf{d}_{12}\right) & =y_{1}\left(t_{1}, d_{1}\right) \circ y_{2}\left(t_{2}, d_{2}\right) \\
& =R_{s}\left(\tau, \mathbf{d}_{12}\right) * R_{h p}\left(\tau, \mathbf{d}_{12}\right) * R_{f}\left(\mathbf{r}_{\perp}\right) \\
& =R_{s p}\left(\tau, \mathbf{d}_{12}\right) * R_{f}\left(\mathbf{r}_{\perp}\right)
\end{aligned}
$$

In this representation, '。' denotes the correlation operation, $\tau$ is the time delay between two signals, $\mathbf{d}_{12}$ is the space vector between two hydrophones, $\mathbf{r}_{\perp}$ is the position vector in the horizontal lag plane, $R_{s}\left(\tau, \mathbf{d}_{12}\right)$ is the autocorrelation function of the transmitted signal, $R_{h p}\left(\tau, \mathbf{d}_{12}\right)$ is the cross-correlation function of the backscattered impulse response from a plane interface, $R_{f}\left(\mathbf{r}_{\perp}\right)$ is the autocorrelation function of the probability density function of the sea-bottom interface roughness, $R_{s p}\left(\tau, \mathbf{d}_{12}\right)$ is the cross-correlation function of the backscattered echoes from a plane interface. The relationship between the correlation operation and the convolution operation is used in the formula derivation. If there is no time delay between the two signals, then:

$$
\begin{aligned}
R\left(\mathbf{d}_{12}\right) & =R_{s}\left(\mathbf{d}_{12}\right) * R_{h p}\left(\mathbf{d}_{12}\right) * R_{f}\left(\mathbf{r}_{\perp}\right) \\
& =R_{s p}\left(\mathbf{d}_{12}\right) * R_{f}\left(\mathbf{r}_{\perp}\right)
\end{aligned}
$$

It indicates that the spatial correlation function of 
sea-bottom reverberation can be expressed as the convolution between the spatial correlation function of the reverberation backscattered by a plane interface and the autocorrelation function of the probability density function of the sea-bottom interface roughness. There is a relationship between $R_{f}\left(\mathbf{r}_{\perp}\right)$ and $S(\boldsymbol{\omega})$ :

$$
R_{f}\left(\mathbf{r}_{\perp}\right)=\frac{1}{2 \pi \sigma^{2}} \int_{-\infty}^{\infty} S(\boldsymbol{\omega}) e^{i \omega \mathbf{r}_{\perp}} d \boldsymbol{\omega}
$$

where $\boldsymbol{\omega}=\left(\omega_{x}, \omega_{y}\right)$.

According to the above derivation, inverse Fourier transform and convolution properties, the spatial correlation function of sea-bottom reverberation is related to the interface roughness of the sea-bottom. The isotropic interface roughness of the sea-bottom leads to a circular planform of the correlation function whose width varies with roughness. The weaker the roughness spectrum, the wider the width. The anisotropic interface roughness of the sea-bottom leads to an elliptical planform of the correlation function whose major axis in the direction of weaker roughness.

\section{Simulation and analysis}

In order to verify the effect of the change of sea-bottom roughness on the spatial correlation characteristics, combined with the actual application environment of the acoustic correlation sonar, we have simulated the sea-bottom reverberation and correlation function under certain conditions[16]. The time-dependent model of the acoustic intensity backscattered by the sea-bottom proposed by Sternlicht and Moustier is used to calculate the sea-bottom reverberation, but the sediment volume backscatter in this model is ignored[17].In the case of isotropic sea-bottom roughness, we use the Jackson model to calculate the backscattering cross section changing with incident angle[18]. In the case of anisotropic sea-bottom roughness, we use the Premus model to calculate the backscattering cross section changing with azimuth angle[14].

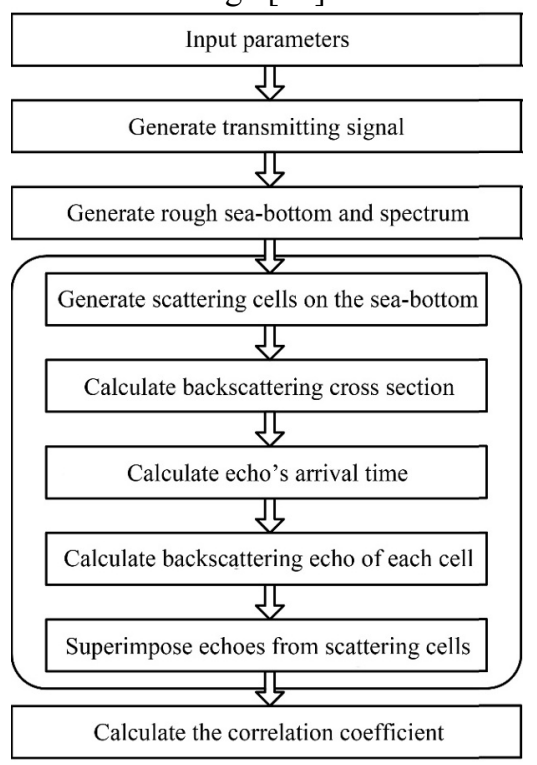

Fig. 3. Simulation flow chart.
Some settings and parameters in the simulation process are as follows: (1)There are one transmitting transducer and eight hydrophones. The eight hydrophones form a sparse matrix, and the spatial spacing between the hydrophones forms the sampling point of the correlation function. The shape of the correlation function can be determined according to the fitting of the theoretical and data's correlation function. (2)The transmitting sound wave is the broadband pseudorandom signal modulated by the carrier wave. The frequency of the carrier wave is $23 \mathrm{kHz}$, and the sampling frequency of the sea-bottom reverberation is $97.75 \mathrm{kHz}$. The equivalent synthetic beamwidth of the system is $30^{\circ}$. The velocity of sound in the seawater is $1528 \mathrm{~m} / \mathrm{s}$. The sea-bottom depth is $100 \mathrm{~m}$. There is no time delay between two signals for correlation. (3)The data's correlation function under the same sea-bottom roughness condition is calculated 50 times and averaged as the final result to eliminate the random error. The simulation process is shown in Fig. 3.

\subsection{Influence of isotropic roughness}

Under the isotropic interface roughness of sea-bottom, the backscattering cross section changing with incident angle can be calculated by the following formula:

$$
\sigma(\theta)= \begin{cases}\frac{g^{2}(0)}{8 \pi \sin ^{2} \theta \cos ^{2} \theta} \int_{-\infty}^{\infty} \exp \left(-q u^{2 \alpha}\right) \mathrm{J}_{0}(u) u d u & \theta>0 \\ \frac{g^{2}(0)}{8 \pi \alpha} C_{h}^{2} 2^{1-2 \alpha}\left(2 k^{2}\right)^{(\alpha-1) / \alpha} \Gamma\left(\frac{1}{\alpha}\right) & \theta=0\end{cases}
$$

where

$$
\begin{aligned}
\alpha & =\frac{\gamma_{2}}{2}-1 \\
C_{h}^{2} & =\frac{2 \pi w_{2} h_{0}^{-\gamma_{2}} \Gamma(2-\alpha) 2^{-2 \alpha}}{\alpha(1-\alpha) \Gamma(1+\alpha)} \\
q & =C_{h}^{2} \cos ^{2} \theta \sin ^{-2 \alpha} \theta 2^{1-2 \alpha} k^{2(1-\alpha)}
\end{aligned}
$$

$g(0)$ is the reflection coefficient of the sound wave at the interface, $\Gamma(\cdot)$ is the Gamma function, $\mathrm{J}_{0}(\cdot)$ is a zero-order Bessel function of the first kind. The backscattering cross section of the sea-bottom reverberation is shown in Fig.4. The backscattering cross section gradually decreases with the increase of the incident angle, and does not change with the azimuth angle. The horizontal spatial correlation of the sea-bottom reverberation is shown in Fig.5. The planform of the correlation function is circular which means that the correlation coefficient of the sea-bottom reverberation decreases in all directions at the same speed.

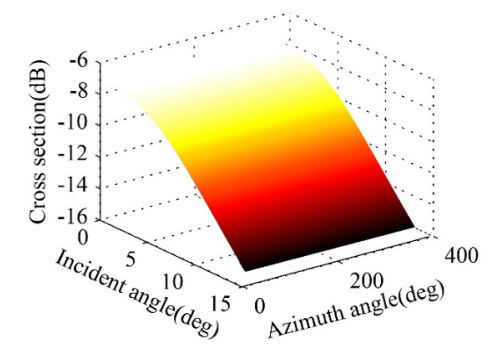


Fig. 4. Backscattering cross section. The spectrum intensity is $1 \times 10^{-10}$, the spectrum exponent is 3.4 .

The spectral intensity and the spectral exponent are two important parameters of the seafloor roughness. In Fig.6 and Fig.7, we can find that the width of the correlation function (defined as the corresponding width when the correlation coefficients drop to 0.707) gradually decreases with the increase of them, that means, the correlation between the received signals decreases, though the spacing of hydrophones remains the same.

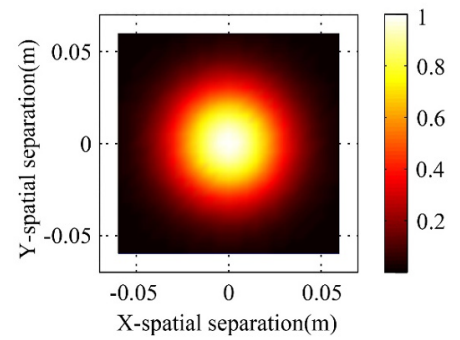

Fig. 5. Horizontal spatial correlation. The parameters are the same as Fig.4.

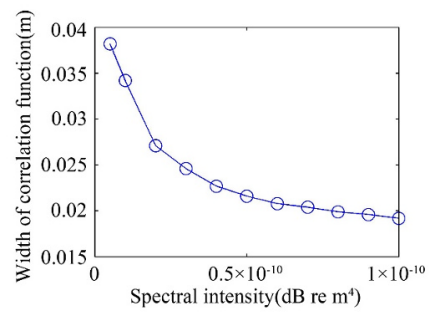

Fig. 6. The width of correlation function. The spectral exponent is 3.4 .

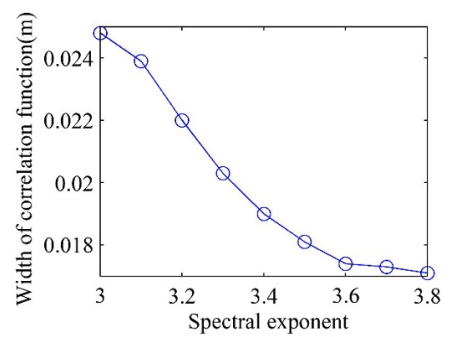

Fig. 7. The width of correlation function. The spectral intensity is $1 \times 10^{-10}$.

\subsection{Influence of anisotropic roughness}

According to the model for the spatially covariant field scattered by randomly rough pressure-release surfaces [13]

$$
\begin{aligned}
\Gamma_{12}= & \frac{A k^{2} f_{1}(\Theta) f_{2}(\Theta) e^{i k\left(r_{1}-r_{2}\right)}}{8 \pi^{2} r^{\prime 2} r_{1} r_{2}} e^{-\Gamma} e^{-\left(k^{2} / 8\right)\left(\bar{\alpha}^{2} U^{2}+\bar{\beta}^{2} V^{2}\right)} \\
& \times \int_{-\infty}^{\infty} D e^{i k\left(\mathbf{r}_{\perp} \cdot \overline{\mathbf{s}}\right)} e^{G R_{f}\left(\mathbf{r}_{\perp}\right)} d \mathbf{r}_{\perp}
\end{aligned}
$$

where $A, U, V$ are the parameters related to the beam footprint, $r$ is the distance from the source to the origin of the scattering cell, $D$ is a Gaussian illumination function introduced to suppress the edge effects, $\bar{\alpha}, \bar{\beta}, \bar{S}, G, \Gamma, f_{1}(\Theta), f_{2}(\Theta)$ are related to the relative position of the hydrophone and the sea-bottom. The above formula can be used to calculate the backscattering intensity at different azimuth angle under the anisotropic sea-bottom roughness[14]

$$
\Gamma\left(\mathbf{K}_{2}\right)=\frac{A k^{2} f^{2}(\theta) e^{-G}}{8 \pi^{2} r^{4}} \int_{-\infty}^{\infty} D e^{i k\left(\mathbf{r}_{\perp} \cdot \mathbf{K}_{2}\right)} e^{G R_{f}\left(\mathbf{r}_{\perp}\right)} d \mathbf{r}_{\perp}
$$

and the backscattering cross section:

$$
\sigma=\Gamma\left(\mathbf{K}_{2}\right) r^{2} / a
$$

where $\mathbf{K}_{2}$ represents the incident angle and the azimuth angle of the scattering cell, $a$ is the area of the scattering cell. When the roughness spectrum is more intensive at $45^{\circ}$, the backscattering cross section of the sea-bottom reverberation is shown in Fig.8. It is related not only to the incident angle but also to the azimuth angle of scattering cell. The backscattering cross section is larger in the direction of more intensive sea-bottom roughness, which means that the reverberation backscattered from sea-bottom in this direction is more intensive. This can be verified by the acoustic seafloor scattering and penetration experiments as part of the Seafloor Acoustic Experiment-1999(SAX99)[19]. As the incident angle increases, the influence of the seafloor roughness on the backscattering cross section increases gradually which brings about more intensive anisotropy in the backscattering cross section. This is understandable, the sound wave is close to normal incidence at all azimuth angles when the incident angle is small, and the anisotropy of the sea-bottom roughness has little effect on it. The horizontal spatial correlation of the sea-bottom reverberation is shown in Fig.9. In the direction of more intensive sea-bottom roughness, the correlation of sea-bottom reverberation reduces more rapidly as the spacing of the hydrophones increases. Although more intensive sea-bottom roughness is helpful to intensify the reverberation, but it weakens the correlation.

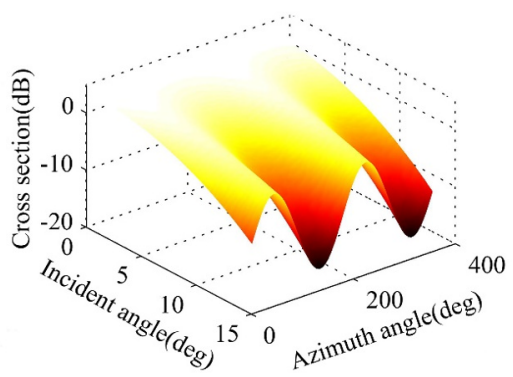

Fig. 8. Backscattering cross section for the parameters of sea-bottom roughness $\sigma=0.0334, \alpha=0.97, \varphi=45^{\circ}, d=0.3$.

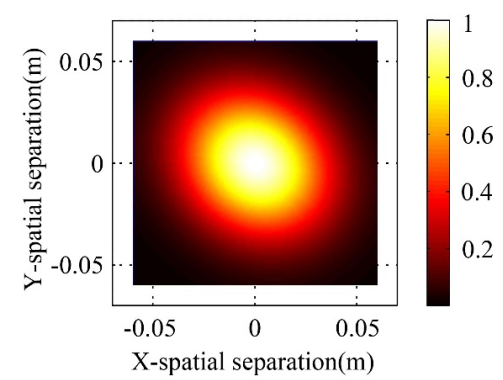

Fig. 9. Horizontal spatial correlation. The parameters are the same as Fig.8.

The direction in which the more intensive sea-bottom roughness concentrates is adjusted to $135^{\circ}$, the influences of the sea-bottom roughness on the 
backscattering cross section and the horizontal spatial correlation are shown in Fig.10 and Fig.11. Other parameters remain unchanged, as we can see, the backscattering cross section is also unchanged when the incident angle is $0^{\circ}$, the backscattering cross section is larger in the direction of the more intensive sea-bottom roughness when the incident angle is $15^{\circ}$. Also in this direction, the correlation reduces more rapidly. This is consistent with the above result.

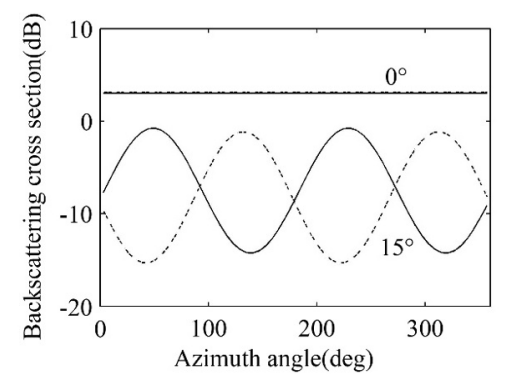

Fig. 10. Backscattering cross section. $\varphi=45^{\circ}$ : solid line, $\varphi=135^{\circ}$ : dashed line, other parameters are the same as Fig. 8 .

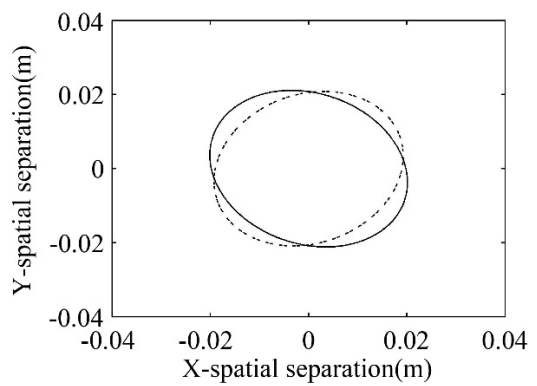

Fig. 11. Horizontal spatial correlation. $\varphi=45^{\circ}$ : solid line, $\varphi=135^{\circ}$ : dashed line, the correlation coefficients on the ellipse are 0.707 , and other parameters are the same as Fig.8.

\section{Conclusions}

High-precision sea-bottom interface is difficult to obtain, this paper uses fractal methods to generate isotropic and anisotropic rough sea-bottom for the simulation of the sea-bottom reverberation and the correlation function. In the simulation, the horizontal spatial correlation is weakened as the enhancement of the isotropic roughness spectrum. The influence of the anisotropic sea-bottom roughness on the horizontal spatial correlation is reflected in the anisotropic correlation function, the correlation is weaker in the direction of the more intensive sea-bottom roughness. The relationship between the horizontal spatial correlation of the sea-bottom reverberation and the sea-bottom roughness is also derived. The conclusion of the formula derivation and the simulation is consistent. The sea-bottom roughness affects the synthesis of the reverberation through the backscattering cross section of the scattering cell, which in turn affects the horizontal spatial correlation. It should be noted in this paper that the wavelength of the anisotropic part of the sea-bottom roughness is much larger than the wavelength of the carrier wave, but it does not form a sea-bottom slope in the beam footprint. The horizontal spatial correlation is usually anisotropic, this is related to the sea-bottom slope[20] and the anisotropic sea-bottom roughness. In the high-frequency sea-bottom acoustics, the acoustic scattering from the sea-bottom interface is dominant while the sediment volume scattering cannot be ignored in many cases[21]. The effect of the sedimentary properties on the horizontal spatial correlation needs further investigation.

\section{References}

1. F. R. Dickey, Jr and J. A. Edward. Proceedings of IEEE Plans, 17, 269-279 (1978)

2. S. K. Hole, B. Woodward, W. Forsythe. IEEE J. Ocean. Eng., 17, 269-279 (1992)

3. A. Keary, M. Hill, P. White, et al. Int. Symp. on Unmanned Untethered Submersible Technology, 446-454 (1999)

4. W. Zhu, F. Lei, C. Wang, et al. Acta Acustica, 36, 338-346 (2006)

5. K. B. Briggs. IEEE J. Ocean. Eng., 14, 360-367 (1989)

6. K. B. Briggs, D. Tang, K. L. Williams. IEEE J. Ocean. Eng., 27, 505-514 (2002)

7. A. P. Lyons, W. L. J. Fox, T. Hasiotis, et al. IEEE J. Ocean. Eng., 27, 515-524 (2002)

8. A. P. Lyons, D. C. Brown. IEEE J. Ocean. Eng., 38, 91-97 (2013)

9. D. R. Jackson, M. D. Richardson, K. L. Williams, et al. IEEE J. Ocean. Eng., 34, 407-422 (2009)

10. D. R. Jackson, M. D. Richardson. High-frequency seafloor acoustics. (Springer, New York, 2007)

11. N. Ma, D. Vray, P Delachartre, et al. Ultrasonics Symposium, 2, 1077-1080 (1995)

12. B. V. Smith, P. R. Atkins. J. Acoust. Soc. Am., 89, 2197-2206 (1991)

13. J. M. Restrepo, S. T. Mcdaniel. J. Acoust. Soc. Am., 87, 2033-2043 (1990)

14. V. Premus, D. J. Acoust. Soc. Am., 96, 2887-2896 (1994)

15. B. Pesquet-Popescu, J. L. Vehel. Signal Processing Magazine IEEE, 19, 48-62 (2002)

16. D. Kuai, C. Wang, L. Feng, et al. Acta Acustica, 34, 385-395 (2009)

17. D. D. Sternlicht, C. P. Moustier. J. Acoust. Soc. Am., 114, 2709-2725 (2003)

18. D. R. Jackson, D. P. Winebrenner, A. Ishimaru. J. Acoust. Soc. Am., 79, 1410-1422 (1986)

19. M. E. Richardson, K. B. Briggs, A. Lyons, et al. Proc. Inst. Acoust., 23, 383-390 (1999)

20. S. Yan, W. Qiu, E. Zhao, et al. Acta Acustica. (to be published)

21. D. Li, C. Wang, E. Zhao, et al. Acta Acustica, 40, 198-206 (2015) 